\title{
Urinary antigene and PCR can both be used to detect Legionella pneumophila in children's hospital-acquired pneumonia
}

\author{
Sayed-Yousef Mojtahedi (1), Aliakbar Rahbarimanesh (2), Samileh Noorbakhsh (3), \\ Hossein Shokri (4), Saeedreza Jamali-Moghadam-Siyahkali (5), Anahita Izadi (6)
}

(1) Department of Pediatric Nephrology, Bahrami Hospital, Tehran University of Medical Sciences, Tehran, Iran; (2) Department of Pediatric Infectious Disease, Bahrami Hospital, Tehran University of Medical sciences, Tehran, Iran; (3) Research Center of Pediatric Infectious Disease, Iran University of Medical Sciences, Tehran, Iran; (4) Bahrami Hospital, Tehran University of Medical Sciences, Tehran, Iran; (5) Ziaeian Hospital, University of Medical Sciences, Tehran, Iran; (6) Department of Pediatric Infectious disease, Tehran University of Medical science, Tehran, Iran

This article is distributed under the terms of the Creative Commons Attribution Noncommercial License (CC BY-NC 4.0) which permits any noncommercial use, distribution, and reproduction in any medium, provided the original author(s) and source are credited.

\begin{abstract}
Legionella pneumophila is the causative agent of more than $95 \%$ cases of severe Legionella pneumonia. Nosocomial pneumonias in different hospital wards is an important medical and pharmaceutical concern. This study aimed to detect Legionella with two methods: polymerase chain reaction (PCR) and detection of urine antigenic test (UAT) in patients suffering from nosocomial pneumonia admitted to pediatric intensive care unit (PICU) of children hospitals. This study was conducted in PICU wards of Rasool Akram and Bahrami children hospitals, Tehran, Iran during 2013 - 2014. In patients diagnosed with hospital-acquired pneumonia, intratracheal secretion samples for PCR and urine sample for UAT were taken. Simultaneously, PCR and urinary antigen test were conducted using commercial kits. The results of urinary antigen test and PCR were analyzed by SPSS v. 19 for statistical comparison. In this study, 96 patients aging 2.77 years on average with two age peaks of less than 1 year and 7-8 year were enrolled. More than half of the patients were under 1 year old. The most common underlying diseases were seizure, Acute Lymphoblastic Lymphoma, Down syndrome and metabolic syndromes. The positivity rate of Legionella urinary antigen test was $16.7 \%$ and positivity rate of PCR test was $19.8 \%$. There were no significant associations between the results obtained by both assays with age, gender or underlying diseases. In conclusion, PCR is a better detection method for Legionella infection than urinary antigen test, but the difference between the two methods was not significant.
\end{abstract}

Key Words: Legionella pneumonia, Polymerase chain reaction, Urinary antigen test, hospitalacquired pneumonia, pediatric intensive care unit.

Eur J Transl Myol 29 (2): 112-117, 2019

Legionella are Gram-negative, aerobic bacteria residing in aquatic environments as an intracellular parasite of free-living amoeba which can cause varying degrees of respiratory infections in communities, hospitals and health-care-retirment homes. ${ }^{1}$ Legionellaceae family contains over 50 species and 64 serogroups. Legionella Pneumophila species cause approximately $90 \%$ of Legionella infections, ${ }^{2}$ and Legionnaires' diseases and of nosocomial pneumonia., ${ }^{1,2}$ Serogroup 1 of Legionella Pneumophila is considered to be responsible for the majority of the reported cases of Legionella infection. ${ }^{3}$
Approximately 1 to 30 percent of hospital-acquired pneumonias arise from Legionella. ${ }^{1,2}$ Mortality rate varies in different patients and this rate is higher in elderly and immunocompromised people in whom 80 percent mortality rate is reported. ${ }^{3}$ One of the peculiar features of this bacterium is hardly staining. Thus, base fusin is recommended instead of safranin for its staining. Isolation and culture of this bacterium is possible in charcoal yeast extract selective medium. ${ }^{4}$ This bacteria can act in sporadic and epidemic forms, simultaneously. On the other hand, simultaneous infection of Legionella with other respiratory pathogenic agents such as 
streptococcus pneumonia, haemophilus influenza and mycobacterium tuberculosis can lead to more complicated health problems. Patients suffering from Legionella do not have serious risks if they diagnosed and treated in the initial phase of the infection. Treatment course lasts 10-14 days. ${ }^{5}$ People with normal immune system show good response to therapy while treatment in immunocompromised patients have delayed or even failured treatment and diagnosis. ${ }^{6}$ Bronchial aspiration is the best candidate specimens for diagnostic which have approximately $90 \%$ of sensitivity, while sputum specimens show sensitivity around 70 percent. $^{7}$ Generally, bacterial culture, direct fluorescent antibody staining and tracking antigen in urine are used to indentify Legionella in clinical samples. ${ }^{8}$ The gold standard method for diagnosing Legionella is bacterial culture, but delayed incubation time and the negative effect in culture when antibiotic therapy is present, are disadvantages of the method. Bronchoalveolar Lavage (BAL) samples can be stained with Giemsa solution and check with an optical microscope; but direct fluorescent antibody test is more accurate with a sensitivity of $70 \%$. Newer assays have been developed for identification of Legionella pneumophila soluble antigens in urine which show 75 to 90 percents of sensitivity. ${ }^{9,10}$ A comprehensive and complete diagnostic method with higher sensitivity rate, capable of diagnosing the initial disease phases and asymptomatic patients, is essential. In this study we compared the PCR and urinary antigen methods to detect Legionella pneumophila in hospitalized patients.

\section{Materials and Methods}

This cross-sectional study was conducted in pediatric intensive care unit (PICU) wards of Rasool Akram hospital complex and Bahrami hospital in Tehran through 2013 to 2014. Intratracheal secretion samples for PCR and urine sample for urinary antigen test were taken from patients with diagnosed hospital-acquired pneumonia. Samples were collected in sterile tubes and stored in -20'C until analysis. Urinary antigen test was performed with Alere CORIS BioConcept ${ }^{\circledR}$ Legionella Urinary Antigen Card. Real time PCR investigations were executed base on the manufacturer's instructions in order to These PCR tests were run for detection of the 16s ribosomal gene in L. pneumophila species. The sequence of the forward primer was 5'GTTAAGTCCCGCAACGA-3' from GenBank entry N315 (NC-002745), positions 1100 to 1116 . The sequence of the reverse primer was 5'AGGAGGTGATCCAGCC-3', from GenBank entry N315 (NC-002745), positions 1551 to 1536.The PCR was performed on a model 9600 GeneAmp PCR system (Applied Biosystems). $10 \mu \mathrm{L}$ of RT-PCR Master mix, 1 $\mu \mathrm{L}$ of the primer/probe, $4 \mu \mathrm{L}$ of distilled water free of RNAse/DNAse and $5 \mu \mathrm{L}$ of the samples from patients were added to each reaction. After 10 minutes of denaturation at $95^{\circ} \mathrm{C}$, Polymerase chain reaction was run for 35 cycles at $90^{\circ} \mathrm{C}$ for 20 seconds and $65^{\circ} \mathrm{C}$ for 50 seconds. The results of urinary antigen test and PCR were analyzed by SPSS v.19 for analytical and descriptive comparisons.

\section{Results}

In this study 96 patients were evaluated which $52.1 \%$ of them were females. The patients' had an age with an average $2.77 \pm 2.72$, of which $56.2 \%$ had an age lower than 1 year age. Two age peaks were observed. The first peak was under 1 year age $(32 \%)$ and the other one was between $7-8$ years of age $(19 \%)$. The most common underlying diseases were seizure $(n=24)$, acute lymphoblastic leukemia (ALL) $(n=12)$, Down syndrome $(n=10)$ and metabolic syndrome $(n=8)$ respectively. The remaining 45 patients were affected with other diseases presented in table 1 . There was no association between occurrence of underlying disease with age $(\mathrm{p}=0.338)$ or gender $(\mathrm{p}=0.710)$. Legionella urine antigen test was positive in 16 cases $(16.7 \%)$ whereas PCR was positive in 19 patients (19.8\%). All positive cases in urinary antigen test were also positive for PCR. No significant association was found between urinary antigen test/PCR positivity with age, gender and underlying diseases, but there was a trend toward a strong association in the case of "drowning". There 6 cases of drowning, which 2 cases of them were positive for both urinary antigen test and PCR $(33.3 \%)$ but this figure didn't reach statistical significance $(\mathrm{p}=0.09)$. 12 cases $(12.5 \%)$ acquired pneumonia after initiating ventilation while 84 cases (87.5\%) had pneumonia before ventilation. Out of 12 post-ventilation pneumonia cases, 3 cases occurred less than 5 days after ventilation (25\%) while 9 other pneumonia cases appeared after 5 days of ventilation. There was a strong association between the time of onset of post-ventilation pneumonia and disease course. The mortality rate in after-5-days pneumonia patients (6 out of 9) was significantly higher than the other group of patients ( 0 out of 3$) \quad(\mathrm{P}<0.0001)$. The final clinical outcome displayed death in 12 cases $(12.5 \%)$, while the remaining patients were discharged either without complications $(55.2 \%)$ or with some health complications $(32.3 \%)$. There was no significant association between disease course with gender $(\mathrm{p}=0.223)$, age $(\mathrm{p}=0.157)$, urinary antigen test positivity $(\mathrm{p}=0.162)$ or PCR positivity $(\mathrm{p}=0.728)$. Overally, the most common symptoms of patients were respiratory distress in 51 cases followed by abnormal X-ray in 41 cases and cyanosis in 26 cases.

\section{Discussion}

Our patients could be categorized into two age peaks. The first peak in our study was similar to Huoug Ple et al. study, ${ }^{11}$ in which they concluded that the highest rate of atypical pathogens pneumonia occurs in patients under 2 years of age. The second peak in our study was not reported in any other study. The disease complications in these patients were milder than those in the first age peak, 
Urinary antigene and PCR for Legionella pneumophila

Eur J Transl Myol 29 (2): 112-117, 2019

Table 1. Basic Characteristics of the study

\begin{tabular}{|c|c|c|c|c|c|c|}
\hline \multirow{2}{*}{$\begin{array}{l}\text { Underlying } \\
\text { condition }\end{array}$} & \multirow{2}{*}{$\begin{array}{c}\text { No. of } \\
\text { patienrs } \\
\text { (Percent) }\end{array}$} & \multirow{2}{*}{$\begin{array}{c}\text { No. of } \\
\text { positive } \\
\text { PCR cases }\end{array}$} & \multirow{2}{*}{$\begin{array}{c}\text { No. of } \\
\text { positive } \\
\text { Urinary } \\
\text { Antigen test } \\
\text { cases }\end{array}$} & \multirow[t]{2}{*}{ Age Interval (Mean) } & \multicolumn{2}{|c|}{ Gender } \\
\hline & & & & & Male & Female \\
\hline Cardiomyopathy & $4(4.16 \%)$ & 1 & 1 & $\begin{array}{c}2 \mathrm{mo}-11 \mathrm{mo} \\
(5.3 \mathrm{mo})\end{array}$ & 1 & 3 \\
\hline SMA & $1(1.04 \%)$ & 0 & 0 & 8 years & 0 & 1 \\
\hline Hypoglycemia & $3(3.12 \%)$ & 0 & 0 & $\begin{array}{c}2 \text { mo }-11 \text { years }(5.8 \\
y r)\end{array}$ & 2 & 1 \\
\hline LOC decrease & $5(5.2 \%)$ & 1 & 0 & $\begin{array}{c}6 \text { mo }-9 \text { years }(4.5 \\
y r)\end{array}$ & 4 & 1 \\
\hline Poor Feeding & $3(3.12 \%)$ & 0 & 0 & $3 \mathrm{mo}$ & 2 & 1 \\
\hline Myopathy & $3(3.12 \%)$ & 0 & 0 & $\begin{array}{c}6 \mathrm{yr}-8 \mathrm{yr} \\
(6.9 \mathrm{yr})\end{array}$ & 3 & 0 \\
\hline ALL & $\begin{array}{c}12 \\
(12.48 \%)\end{array}$ & 3 & 3 & $\begin{array}{c}2 \mathrm{yr}-10 \mathrm{yr} \\
(7.3 \mathrm{yr})\end{array}$ & 4 & 8 \\
\hline Asphyxia & $2(2.08 \%)$ & 0 & 0 & $45 \mathrm{~d}-2 \mathrm{mo}(1.7 \mathrm{mo})$ & 0 & 2 \\
\hline $\mathrm{CP}$ & $3(3.12 \%)$ & 1 & 1 & $\begin{array}{c}2 \mathrm{yr}-7 \mathrm{yr} \\
(5 \mathrm{yr})\end{array}$ & 3 & 0 \\
\hline Seizure & $\begin{array}{c}26 \\
(27.08 \%)\end{array}$ & 5 & 4 & $3 \mathrm{mo}-11 \mathrm{yr}(6.9 \mathrm{yr})$ & 11 & 15 \\
\hline Drowning & $4(4.16 \%)$ & 2 & 2 & $\begin{array}{c}4 \mathrm{yr}-10 \mathrm{yr} \\
(8.1 \mathrm{yr})\end{array}$ & 2 & 2 \\
\hline Down Syndrome & $10(10.4 \%)$ & 1 & 0 & $5 \mathrm{mo}-10 \mathrm{yr}(6.9 \mathrm{yr})$ & 4 & 6 \\
\hline Metabolic Disease & $8(8.32 \%)$ & 3 & 3 & $\begin{array}{c}3 \mathrm{mo}-6 \mathrm{yr} \\
(4.5 \mathrm{yr})\end{array}$ & 5 & 3 \\
\hline Gastroentritis & $3(3.12 \%)$ & 1 & 1 & $1 \mathrm{yr}-8 \mathrm{yr}$ & 2 & 1 \\
\hline
\end{tabular}

Note: SMA (Spinal Muscular Atrophy); LOC (level of conciousness); ALL (Acute Lymphoblastic Lymphoma); CP (Cerebral Palsy); GERD (Gastro-esophageal reflux disease); HLH (Hemophagocytic Lymphohistiocytosis)

which may occur due to higher pathogens exposure in community by entrance of children to school. Our data shows that the most common underlying conditions of hospital-acquired pneumonias are seizure, ALL, Down syndrome and metabolic syndromes. Other studies do not support our findings, ${ }^{12,13}$ but this apparent discrepancy may be due to higher rate of pneumonia attributed to aspiration in epilepsy and seizure. On the other hand, ALL found to be one of the most common underlying conditions of hospital-acquired pneumonias, probably because of defect in immunity system and general predisposition to infections. Legionella pneumophila has 
been reported as the cause of $7.22 \%$ of severe atypical pneumonias. ${ }^{1}$ The prevalence of pneumonias due to chlamidya pneumonia and Legionella is rapidly increasing. Considering the importance of this organism in pneumonia pathogenesis, its early identification is important for rapid and accurate therapeutic interventions. In the present study we employed UAT and PCR methods to detect Legionella species in patients with hospital-acquired pneumonia. The traditional gold standard method for identification of this organism has been culturing the Legionella. However, culture of Legionella bacteria from clinical samples could be impaired bacause of the long incubation time, and possible inhibition of the bacteria growth in competition with other faster-growing organisms in the culture medium. ${ }^{14}$ These limitations have prompted to use newer methods. One of such methods is Legionella urinary antigen test. Kanavaki et a $1^{15}$ declare that $80 \%$ of patients with Legionella secrete soluble antigens of this microorganism in urine 1 to 3 days after infection. These antigens are detected in urine for at least 42 to 50 days post infection. This assay has been found to be an appropriate method with several advantages, such easy urine sample collection, high volume collection of sample, and the possibility of identify the antigen in urine even after antibiotic therapy. Short time span to have results is a further advantage of the method. ${ }^{16} \mathrm{PCR}$ is a semi-quantitative method which uses to detect DNA sequences based on amplification of targeted section. This method has been widely used to detect Legionella pneumonia. Lim et al. ${ }^{17}$ have conducted a study on 267 patients with pneumonia symptoms which yielded a 3.3\% positivity rate for Legionella urinary antigen test. Similarly, Goudarzi et al. ${ }^{18}$ have reported a $5.7 \%$ rate of positivity by urinary antigen test. However, $18.4 \%$ of the samples tested by Arnouts et al were positive by UAT, a finding similar to our results $(16.7 \%)$. Jonas et al. ${ }^{19}$ conducted PCR and culture on 250 BAL samples. Their results showed that 8 samples were culture and PCRpositive, whereas 6 other samples were PCR-positive, but culture-negative. Wellinghausen et al. ${ }^{20}$ performed Quantitative PCR on 67 water sewage samples collected from different hospital sections which revealed $98.7 \%$ positivity in PCR while this figure in culture was $70.1 \%$. The sensitivity of urinary antigen test was reported to be 86 to $90 \%$ while its specificity reached 100 percent. ${ }^{15}$ Cloud et al. ${ }^{21}$ in a study on 212 samples reported that $100 \%$ of culture-positive samples were PCR-positive and out of 181 culture-negative samples, 12 were PCRpositive leading to specificity of $93 \%$ for PCR. In another study, Wilson et $\mathrm{al}^{22}$ reported $27 \mathrm{PCR}$ positive and only 7 culture positive samples out of 150 patients. Their results together with our findings indicate higher sensitivity of the PCR assay as compared to better specificity of the urinary antigen test. Furthermore, the differences observed between our results and those reported by others might be due to differences in methods of sample collection, clinical conditions of patients, patients' ethnicity, commercial kits and diagnostic materials. Several other studies have stated that the sensitivity of PCR method for L.pneumophila detection is greater or equal to culture method. ${ }^{23-26} \mathrm{We}$ have not been able to culture our samples due to technical shortcomings. This limitation does not allow us to estimate sensitivity and specificity of UAT and PCR techniques. Another finding in our study was relatively higher positivity rate of Legionella pneumophila infection in patients with an history of drowning; this phenomenon can be attributed to Legionella's growth in water environments. We also observed a higher rate of mortality in patients who developed pneumonia 5 days after ventilation compared to those who developed pneumonia within 2 days post-ventilation. Regarding this issue, there was nothing found in the literature, but it can be speculated that patients with more than 5 days intubation are usually more susceptible subjects of concomitant infections and they were discharged from the hospital while they have worse general condition.

In conclusion, the gold standard method to identify Legionella infections is microbial culture that might show false negative results by many causes, including culture condition and microorganism features and properties. These limitations have prompted employment of newer methods. Present study compared Legionella urinary antigen test and PCR to detect the presence of Legionella pneumophila in hospital-acquired pneumonia. PCR method was more sensitive in the detection of Legionella than urinary antigen test; however the difference between these two methods was not statistically significant.

\section{List of acronyms}

ALL - acute lymphoblastic leukemia

BAL - bronchoalveolar lavage

CP - Cerebral Palsy

PICU - pediatric intensive care unit

UAT - urinary antigen test

\section{Authors contributions}

Sayed-Yousef Mojtahedi supervised the implementation of the study and corrected the final version of the manuscript, Aliakbar Rahbarimanesh participated in selecting patients and carrying out the project, Samileh Noorbakhsh participated in selecting patients and carrying out the project, Hossein Shokri cooperated in statistical consultation and designation of the study, Saeedreza Jamali-Moghadam-Siyahkali wrote the draft of manuscript, Anahita Izadi presented the project's original design and she is the corresponding author of the article.

\section{Acknowledgments}

We would like to thank Mr. Mohammadreza Shokri for providing the required data of this research work.

Funding No funding was obtained for this research project. 


\section{Conflict of Interest}

The authors report no conflict of interest.

\section{Ethical Publication Statement}

We confirm that we have read the Journal's position on issues involved in ethical publication and affirm that this report is consistent with those guidelines.

\section{Corresponding Author}

Anahita Izadi, Department of Pediatric Infectious Disease, Bahrami Hospital, Shahid Kiaee Street (Ghasem Abad), Damavand Street, Tehran, Iran. Postal Code: 1641744991. Phone: Tel: +982173013553

Email: dr.anahita.izadi@gmail.com

\section{E-mails of co-authors}

Sayed-Yousef Mojtahedi: drmojtahed@yahoo.com Aliakbar Rahbarimanesh: Rahbarim@sina.tums.ac.ir Samileh Noorbakhsh: samileh_noorbakhsh@yahoo.com Hossein Shokri: doctorshokri@gmail.com Saeedreza Jamali-Moghadam-Siyahkali:

\section{Saeedjamali99@yahoo.com}

\section{References}

1. Palusińska-Szysz $\mathrm{M}$, Cendrowska-Pinkosz $\mathrm{M}$. Pathogenicity of the family Legionellaceae. Archivum Immunologiae et Therapiae Experimentalis 2009;57:279-90. doi: 10.1007/s00005-009-0035-8

2. Phin N, Parry-Ford F, Harrison $T$, et al. Epidemiology and clinical management of Legionnaires' disease. The Lancet Infectious Diseases 2014;14:1011-21. doi: 10.1016/S14733099(14)70713-3

3. Diederen BMW. Legionella spp. and Legionnaires' disease. Journal of Infection 2008;56:1-12. doi: 10.1016/j.jinf.2007.09.010

4. Fields BS, Benson RF, Besser RE. Legionella and legionnaires' disease: 25 years of investigation. Clin Microbiol Rev 2002;15:506-26. doi: 10.1128/CMR.15.3.506-526

5. Marston BJ, Plouffe JF, File TM Jr , et al. Incidence of community-acquired pneumonia requiring hospitalization. Results of a population-based active surveillance Study in Ohio. The CommunityBased Pneumonia Incidence Study Group. Arch Intern Med 1997;157:1709-18. doi: 10.1001/archinte.1997.00440360129015

6. Lim WS, Macfarlane JT, Boswell TC, et al. Study of community acquired pneumonia etiology (SCAPA) in adults admitted to hospital: implications for management guidelines. Thorax 2001;56:296-301. doi: 10.1136/thorax.56.4.296

7. Den Boer JW, Yzerman EP. Diagnosis of Legionella infection in Legionnaires' disease. Eur J Clin Microbiol Infect Dis 2004;23:871-78. DOI: 10.1007/s10096-004-1248-8
8. Socan M, Marinic-Fiser N, Kese D. Comparison of serologic tests with urinary antigen detection for diagnosis of legionnaires' disease in patients with community-acquired pneumonia. Clin Microbiol Infect 1999;5:201-04. doi: 10.1111/j.1469-0691. 1999.tb00124.x

9. Lindsay DS, Abraham WH, Fallon RJ. Detection of mip Gene by PCR for diagnosis of Legionnaires' disease. J Clin Microbiol 1994; 32: 3068-69.

10. Qasem JA, Mustafa AS, Khan ZU. Legionella in clinical specimens and hospital water supply facilities: molecular detection and genotyping of the isolates. Med Princ Pract 2008;17:49-55.

11. Huong Ple T, Hien PT, Lan NT, et al. First report on prevalence and risk factors of severe atypical pneumonia in Vietnamese children aged 1-15 years. BMC Public Health. 2014;14:1304. doi: 10.1186 /1471-2458-14-1304

12. Ditommaso S, Giacomuzzi M, Rivera SR, et al. Virulence of Legionella pneumophila strains isolated from hospital water system and healthcareassociated Legionnaires' disease in Northern Italy between 2004 and 2009. BMC Infect Dis 2014;14:483. doi: 10.1186/1471-2334-14-483

13. Fang GD, Yu VL, Vickers RM. Disease due to the Legionellaceae (other than Legionella pneumophila). Historical, microbiological, clinical, and epidemiological review. Medicine (Baltimore) 1989;68:116-32.

14. Goudarzi H, SeyedJavadi S, Goudarzi M. Detection of Legionella pneumophila by sputum culture, direct immunoflurscence and PCR. Pejouhesh 2011;35:119-24.

15. Kanavaki SK, Koumantakis P, Makarona M, et al. The laboratory diagnosis of Legionnaires' disease in patients with community acquired pneumonia (CAP). Pneumon 2003;16:173-180.

16. Kashuba $\mathrm{AD}$, Ballow $\mathrm{CH}$. Legionella urinary antigen testing: potential impact on diagnosis and antibiotic therapy. Diagn Microbiol Infect Dis 1996;24:129-39. doi: 10.1016/07328893(96)00010-7

17. Lim WS, Macfarlane JT, Boswell TC, et al. Study of community acquired pneumonia aetiology (SCAPA) in adults admitted to hospital: implications for management guidelines. Thorax 2001;56:296-301. doi: 10.1136/thorax.56.4.296

18. Arnouts PJ, Ramael MR, Ysebaert DK, et al. Legionella pneumophila peritonitis in a kidney transplant patient. Scand J Infect Dis 1991;23:11922. doi: $10.3109 / 00365549109023386$

19. Jonas D, Rosenbaum A, Weyrich S, Bhakdi S. Enzyme-linked immunoassay for detection of PCRamplified DNA of Legionellae in bronchoalveolar fluid. J Clin Microbiol 1995;33:1247-52.

20. Wellinghausen N, Frost C, Marre R. Detection of Legionellae in hospital water samples by quantitative real-time LightCycler PCR. Appl 
Environ Microbiol 2001;67:3985-93. doi: 10.1128/AEM.67.9.3985-3993.2001

21. Cloud JL, Carroll KC, Pixton P, et al. Detection of Legionella species in respiratory specimens using PCR with sequencing confirmation. J Clin Microbiol 2000;38:1709-12.

22. Wilson DA, Yen-Lieberman B, Reischl U, et al. Detection of Legionella pneumophila by real-time PCR for the mip gene. J Clin Microbiol 2003;41:3327-30. doi: 10.1128/JCM.41.7.33273330.2003

23. Cloud JL, Carroll KC, Pixton P, et al. Detection of Legionella species in respiratory specimens using PCR with sequencing confirmation. J Clin Microbiol 2000;38:1709-12.

24. Reischl U, Linde HJ, Lehn N, et al. Direct detection and differentiation of Legionellaspp. and Legionella pneumophila in clinical specimens by dual-color real-time PCR and melting curve analysis. J Clin Microbiol 2002;40:3814-17. doi: 10.1128/JCM. 40.10.3814-3817.2002

25. Stolhaug A, Bergh K. Identification and differentiation of Legionella pneumophila and Legionella spp. with real-time PCR targeting the 16S rRNA gene and species identification by mip sequencing. Appl Environ Microbiol 2006;72:6394-8. doi: 10.1128/AEM.02839-05

26. Templeton KE, Scheltinga SA, Sillekens P, et al. Development and clinical evaluation of an internally controlled, single-tube multiplex realtime PCR assay for detection of Legionella pneumophila and other Legionella species. J Clin Microbiol 2003;41:4016-21. doi: 10.1128/JCM.41.9.4016-4021.2003.

Submissions: February 19, 2019

Revision received: April 15, 2019

Acceptance: April 15, 2019 